\title{
Conformational Dynamics of the Dengue Virus Protease revealed by Fluorescence Correlation and Single-Molecule FRET Studies
}

\author{
Christian Götz ${ }^{\mathrm{a}}$, Gerald Hinze ${ }^{\mathrm{a}}$, Andrea Gellert ${ }^{\mathrm{b}}$, Hannah Maus $^{\mathrm{b}}$, Franziska von \\ Hammerstein $^{\mathrm{b}}$, Stefan J. Hammerschmidt ${ }^{\mathrm{b}}$, Luca M. Lauth ${ }^{a, \S}$, Ute A. Hellmich ${ }^{\mathrm{a}, \mathrm{c}, \#}$, Tanja \\ Schirmeister ${ }^{\mathrm{b}}$, Thomas Basché,
}

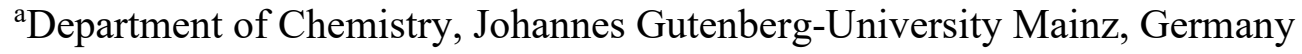
${ }^{\mathrm{b}}$ Institute for Pharmaceutical and Biomedical Sciences, Johannes Gutenberg-University Mainz, Germany

${ }^{\mathrm{c} C e n t r e}$ for Biomolecular Magnetic Resonance (BMRZ), Goethe-University Frankfurt, Germany

\section{S1 Fluorometric assay}

The enzymatic activity of the dye labeled protease was verified with the fluorogenic substrate boc-GRR-AMC in presence and absence of competitive or non-competitive inhibitors. The buffer contained Tris (50 mM, pH 9), CHAPS (1 mM) and optionally glycerol (20 vol\%). Each measurement was performed with a Tecan Infinite F2000 PRO fluorimeter in duplicates in flatbottom 96-well microtiter plates from Greiner Bio-One. A total volume of $200 \mu \mathrm{L}$, containing $180 \mu \mathrm{L}$ buffer, $10 \mu \mathrm{L}$ DMSO as control or inhibitor in DMSO (final concentration $20 \mu \mathrm{M}$ ), $5 \mu \mathrm{L}$ enzyme solution and $5 \mu \mathrm{L}$ substrate (final concentration of $100 \mu \mathrm{M}$ ) was used for each well. The inhibitory activity was compared to the results of the wildtype protease.

\section{S2 Spectroscopic characterization of the FRET pair labeled double mutants of DENV-PR}

Absorption and fluorescence spectra of the FRET pair labeled double mutants of DENV-PR were recorded in assay buffer (50 mM TRIS-HCl pH 9.0, $1 \mathrm{mM}$ CHAPS) with $10 \mathrm{vol} \%$ of DMSO. Notice that for historical reasons, the assay buffer for the measurements on the S79C-G14C double mutant contained 20 vol\% of glycerol, while the assay buffer for the measurements on the other two samples contained no glycerol. Table S1 summarizes the concentrations used.

Table S1: Concentrations of the different samples used for ensemble characterization.

\begin{tabular}{|c|c|}
\hline sample name & concentration / $\boldsymbol{\mu M}$ \\
\hline S79C-G14C & 0.82 \\
\hline S79C-S158C A & 0.95 \\
\hline S79C-S158C B & 0.72 \\
\hline
\end{tabular}


To record the absorption spectra, $600 \mu \mathrm{L}$ of the solutions were filled in a microcuvette. The absorption spectra were recorded at room temperature using a two-beam spectrophotometer (LAMBDA 850 UV/VIS, PerkinElmer, United States).

To record the fluorescence emission spectra, $50 \mu \mathrm{L}$ of the solutions were filled in a precision cuvette. The spectra were measured at room temperature in a 90-degree geometry using a spectrofluorometer (Fluorolog3, Horiba Jobin-Yvon, Germany). For each measurement the spectral bandwidth of the excitation and emission monochromator was set to $1 \mathrm{~nm}$. The absorption and emission spectra are shown in Figure S1.

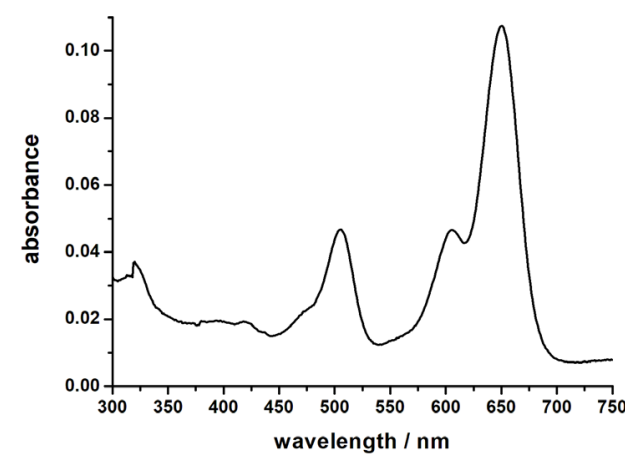

(a)

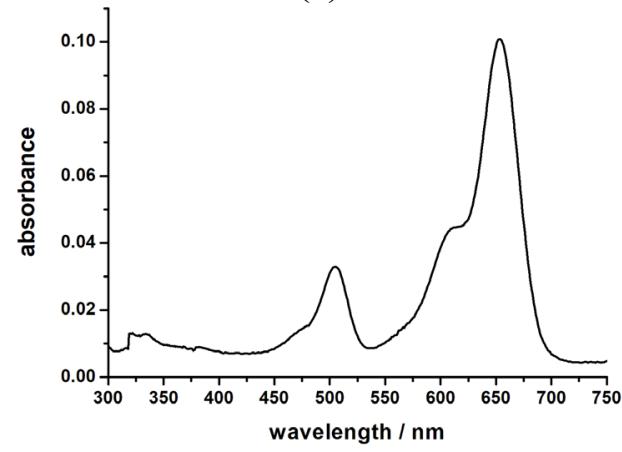

(c)

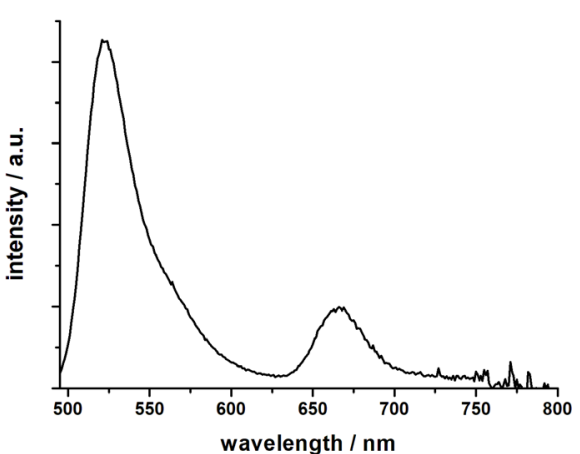

(b)

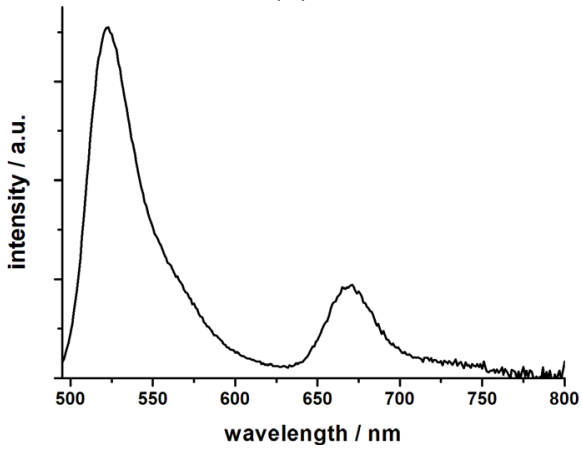

(d)

Figure S1: Absorption and emission spectra, respectively, of the ATTO 488/ATTO 647 N labeled DENV-PR S79C-S158C A $(a, b)$ and the ATTO 488/Cy5 labeled DENV-PR S79C-S158C B (c, d) double mutants, in assay buffer. Following excitation of the donor $(488 \mathrm{~nm})$ emission from both donor and acceptor is observed.

\section{S3 DENV-PR S79C-S158C A: Conformational exchange without and with competitive inhibitor 1}

Following a procedure proposed by Torres and Levitus [Torres, T; Levitus, M. J. Phys. Chem. B 2007, 111, 7392-7400], in Figure $\mathrm{S} 2$ the ratios of the acceptor autocorrelation function $G_{A A}$ and the crosscorrelation function $G_{D A}$ are plotted before and after adding $\mathbf{1}$. Within a simple two-state-model both correlation functions are composed of a diffusional part $T_{i i}$ and a kinetic contribution $X_{i i}$ depending on the kinetic rates $k_{12}, k_{21}$ and the FRET efficiencies in both states $E_{1}$ and $E_{2}$, respectively. Assuming similar diffusion contributions for all correlation functions, $T_{A A}=T_{D A}$, the ratio solely depends on conformational exchange,

$$
\frac{G_{A A}(\tau)}{G_{D A}(\tau)}=\frac{1+a_{A A} \exp \left(-\tau / \tau_{e x}\right)}{1-a_{D A} \exp \left(-\tau / \tau_{e x}\right)}
$$

with $\tau_{e x}=\left(k_{12}+k_{21}\right)^{-1}, a_{A A}=k_{12} k_{21}\left(E_{1}-E_{2}\right)^{2} /\left(k_{21} E_{1}+k_{12} E_{2}\right)^{2}$ 
and $a_{D A}=k_{12} k_{21}\left(E_{1}-E_{2}\right)^{2} /\left[\left(k_{21} E_{1}+k_{12} E_{2}\right)\left[k_{21}\left(1-E_{1}\right)+k_{12}\left(1-E_{2}\right)\right]\right]$. We have performed a fit to the ratio (in absence of the inhibitor) assuming $E_{1}=0.5, E_{2}=1.0$ and a log normal distribution of transition rates. Furthermore, we have accounted for an additional decay in the acceptor auto-correlation caused by triplet kinetics which is absent in the crosscorrelation. We point out that our data evaluation is a first and rough approach, since fitting parameters strongly depend on the chosen model and the method relies on the assumption of $T_{A A}=T_{D A}$. Once the diffusion contribution for the donor decays faster than for the acceptor, the diffusion contribution for the cross-correlation decays faster than for the acceptor, too. As a consequence, besides conformational exchange the ratio shown in Figure S2 might contain some contribution from pure diffusion. This could explain the small increase in the calculated ratio in the presence of inhibitor, the amplitude of which, however, is close to the noise level. In principle, similar ratios could be calculated employing the donor autocorrelation function, however, the donor emission signal is strongly superimposed by donor only species. In addition, any scattering and fluorescing contributions from potential contaminations will be more easily seen in the donor channel, rather than in the acceptor channel.

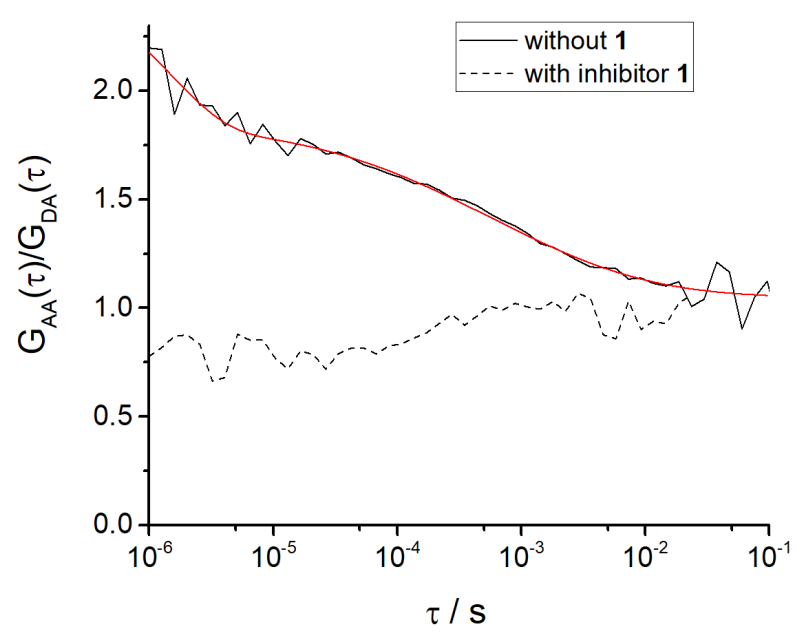

Figure S2: Calculated ratios of the acceptor auto-correlation and the cross-correlation functions without and with inhibitor 1. The red line represents a fit to the data in the absence of inhibitor with a mean exchange time $\left\langle\tau_{e x}\right\rangle \approx 10 \mathrm{~ms}$. The ratio of the mean transition rates $\left\langle k_{12}\right\rangle /\left\langle k_{21}\right\rangle \approx 4.5$ corresponds to the population ratios of the states. That is, the closed conformation appears to be more populated than the open conformation.

\section{S4 DENV-PR S79C-S158C A: Ensemble fluorescence decays before and after adding the competitive inhibitor 1}

Time correlated single photon counting (TCSPC)-experiments at the ensemble level have been performed in solution. In Figure S3 the rise/decay curves of the acceptor emission are plotted after pulsed excitation of the acceptor as well as the donor. In the latter case experiments were performed without and with inhibitor 1. After donor excitation energy transfer to the acceptor leads to a delayed increase of the rise/decay curves compared to direct excitation of the acceptor. All curves were adjusted to the longtime tail solely determined by the acceptor fluorescence lifetime. As can be seen in Figure S3, without inhibitor the rise/decay curve significantly deviates at short times compared to the case of direct excitation, indicating slow (inefficient) energy transfer. The behavior significantly changes after adding $\mathbf{1}$. Now the energy transfer to the acceptor is much more efficient, the longtime tail decay occurring much earlier. 
Note that direct excitation resembles donor excitation with complete (instantaneous) energy transfer.

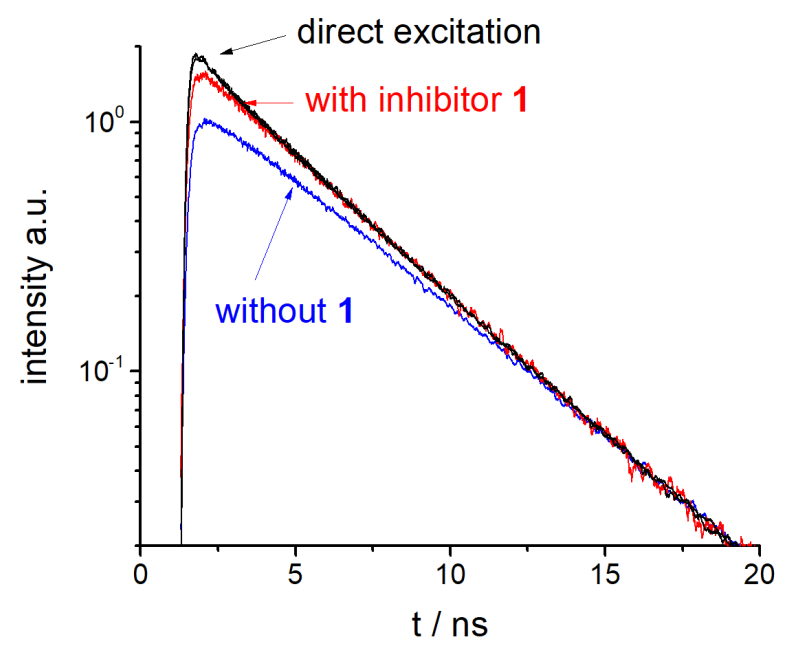

Figure S3: TCSPC-ensemble decay curves of the DENV-PR S79C-S158C A double mutant. For details see text.

\section{S5 DENV-PR S79C-S158C B before and after adding the competitive inhibitor 1}

Figure S4 shows the auto- and cross-correlations before and after addition of 1 . In the absence of the inhibitor, the cross-correlation drops to zero slower than the two autocorrelations. This again indicates that an exchange between different conformations, differing in their FRET efficiency, occurs.

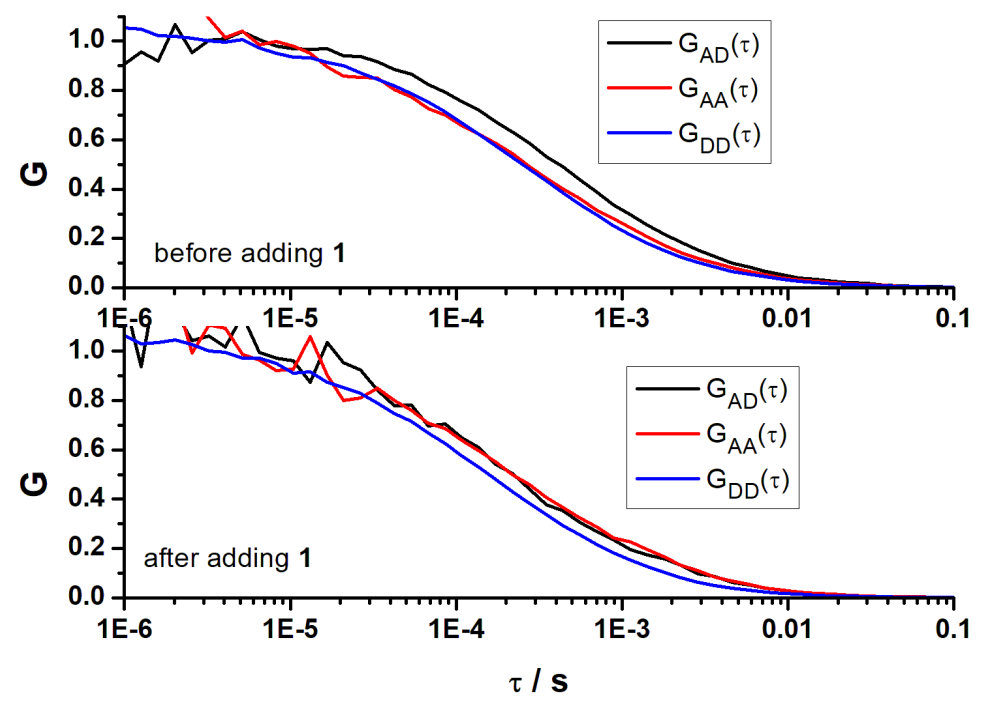

Figure S4: Comparison of the normalized cross-correlation (black curve) with the two normalized auto-correlations (red and blue), each before (upper graph) and after (lower graph) addition of the competitive inhibitor 1 to the ATTO 488/Cy5 FRET pair labeled DENV-PR S79C-S158C double mutant.

Similar to the ATTO 488/ATTO 647N labeled DENV-PR S79C-S158C double mutant (DENV-PR S79C-S158C A) described in the main text, the cross-correlation does not drop to zero slower than the acceptor auto-correlation after addition of $\mathbf{1}$. Once again, this can be interpreted as an inhibitor induced stabilization of a conformation.

Figure S5 shows the 2D histograms obtained from the burst analysis of the intensity time traces before (a) and after (b) addition of $\mathbf{1}$. Again, the population with a FRET efficiency of zero is due to DENV-PR molecules only labeled with the donor dye ATTO 488 and shows a 
significantly longer fluorescence lifetime of the donor. As with the previous studied ATTO 488/ATTO 647N FRET pair labeled DENV-PR S79C-S158C double mutant, the point cloud is significantly elongated along the abscissa before the addition of $\mathbf{1}$. The data deviate characteristically from the static FRET line and are shifted towards longer donor lifetimes, in accordance with the observations described in the main text. Again, this indicates that the DENV-PR, during the transit time through the observation volume, fluctuates between different conformational substates, which differ in their FRET efficiencies. After addition of 1 (see, Figure S5 (b)), the bursts with medium FRET efficiencies which deviated characteristically from the static FRET line disappear almost completely. Solely the population with high FRET efficiencies survives, in good agreement with the static FRET line. This corresponds to the theoretical expectations, since for this double mutant, the distance between the two labeling positions (S79C and S158C) should decrease as a result of the transition from the open to the closed conformation (see Table 1 in the main text), leading to increased FRET efficiencies. The upper parts of Figure S5 (a) and (b) illustrate the corresponding normalized 1D histograms of FRET efficiencies before and after adding 1. Almost exclusively one FRET population at high FRET efficiencies is observed after addition of the competitive inhibitor.
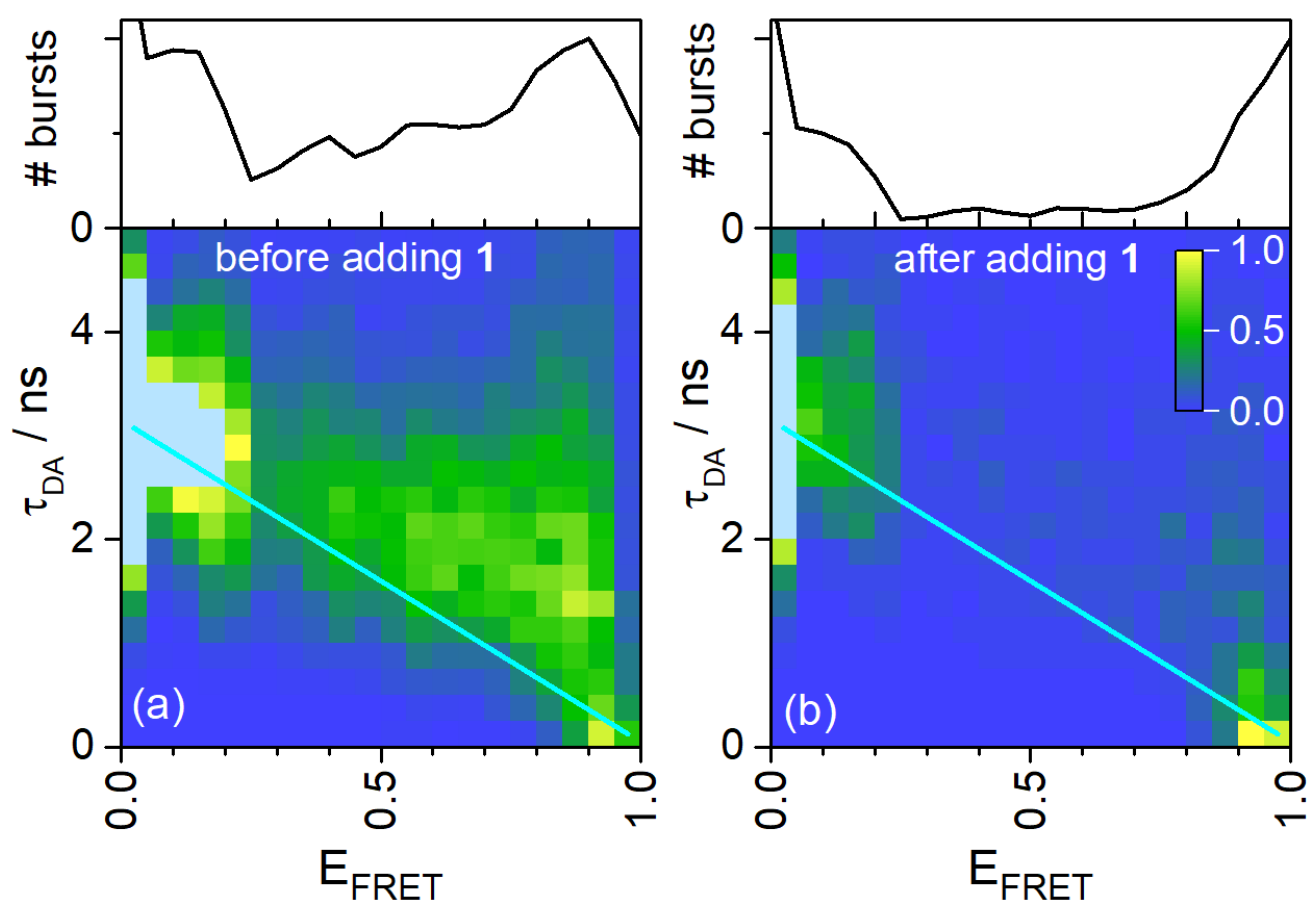

Figure S5: Plot of the normalized occurrences of the individual bursts (individual molecules) within the intensity time trace of the ATTO 488/Cy5 FRET pair labeled DENV-PR S79C-S158C double mutant before (a) and after (b) adding 1 in a $2 \mathrm{D}$ histogram, separated according to donor lifetime $\tau_{D A}$ and FRET efficiency $E_{F R E T}$. For an easier visual comparison of the FRET populations before and after addition of the inhibitor, the respective maxima were normalized to one. The maxima of the donor-only species were not taken into account in the normalization, which is why values greater than one, shown in gray, occur. The cyan lines represent the static FRET lines. The respective normalized 1D histograms of FRET efficiencies are shown as a projection on the top of the $2 \mathrm{D}$ histograms. 